\title{
WHERE DOES STAR FORMATION END IN NGC1058?
}

\author{
ANNETTE FERGUSON AND ROSEMARY WYSE \\ The Johns Hopkins University, Baltimore, MD, IJSA \\ JAY GALLAGHER \\ University of Wisconsin, Madison, WI, USA \\ AND \\ DEIDRE HUNTER \\ Lowell Observatory, Flagstaff, AZ, USA
}

As part of a large project to study the rate, distribution and history of star formation in the outer parts of galactic disks (Ferguson, PhD thesis), we have obtained very deep $\mathrm{H} \alpha$ and B-band images of the nearby face-on spiral NGC1058. Our images reveal extremely low surface brightness outer spiral arms in the B-band which extend well beyond the Holmberg radius and appear to be aligned with underlying HI spiral arms. These features contrast greatly to the flocculent appearance of the innermost regions of the disk and the complete absence of any well defined-arms in the $\mathrm{H} \alpha$ image. The surface brightness profile derived from the B-band image exhibits a sharp fall off in the inner regions of the disk followed by a much shallower decline beyond $\sim R_{25}$ and consequently a single exponential disk cannot be fit to the entire profile.

We have applied the threshold criteria of Kennicutt (1989, ApJ) to the galaxy in order to compare the radial variation of the critical gas density for star formation with the observed gas distribution. NGC1058 provides an excellent test for this model since it is one of the few galaxies for which the radial variation of the gaseous velocity dispersion is known (Dickey, etal., 1990, ApJ). Throughout the disk of NGC1058, we find that the actual gas surface density (atomic plus molecular) lies well below that required for star formation and furthermore that the discrepancy between actual and critical values is most severe in the inner regions of the disk where the bulk of the HII regions are observed (note uncertainties in the rotation curve however). We conclude that the simple threshold model does not appear to explain the observed distribution of star formation in NGC1058. 